\title{
Comparison of Primary Incompatible Schemas with Regard to the Age Gap of Sisters in People with the Major Depressive Disorder with Normal People
}

\author{
Rahim Basaeri $^{1^{*}} \quad$ Neda Alibeigi ${ }^{2}$ \\ 1.University of science and research Branch.Tehran Islamic Azad university.Tehran Iran \\ 2.University of social welfare and rehabilitation Iran Tehran
}

\begin{abstract}
Objective: The purpose of this study was to compare the primary incompatible schemas with respect to age range among people with the major depressive disorder and normal people.Research Method: the post-traumatic (event) research method and the statistical population included two groups of the major depression with normal people in Tehran. Among them, a group of 30 patients chose to refer to psychiatric treatment centers and counseling centers with available sampling method (for groups with the major depressive disorder) and a group of 30 patients' accompaniment (rellies (مخف relatives ) by classification randomly (for the normal group) method. The research tools consisted of a questionnaire of the primary incompatible schemas of Young's short form and questionnaire of the demographic information. Data were analyzed by multivariate analysis of variance (MANOVA).Results: The results of the analysis showed that in all subscales of the primary incompatible schemas, except the subscales of sacrifice, obedience, and magnanimity, were significantly different between the two study groups $(\mathrm{P}<0.01)$. Also, logistic regression analysis showed that 34 to $45 \%$ of the major depressive disorder was explained by the subscales of the primary incompatible schemas.Conclusion: It seems that consideration of such variables plays an important role in the primary and secondary prevention, and it is necessary for psychologists and counselors to pay attention to these variables in clinical and counseling environments.
\end{abstract}

Keywords: The Primary Incompatible Schemas, Age Difference, The major Depressive Disorder.

DOI: $10.7176 /$ RHSS/9-10-09

Publication date:May $31^{\text {st }} 2019$

\section{Introduction}

Depression is currently the fourth most common disease in the world, and according to estimates, it is expected that this disease will be the most common disease in the world by 2020 (Parker and Roy, quoted by Bakhshipoor $\&$ et al., 2015). According to a national study of diseases and injuries in Iran, depression is the third most common health problem in the country (Montazeri \& et al., 2013). The prevalence of the major depressive disorder is estimated to be between 5\% to $17 \%$ and the American Psychiatric Association (2013) reports a 12month prevalence rate of the major depressive disorder in the United States of around 7\%. Sadeqirad \& et al (2010) also they estimated the prevalence of the major depression at $25 \%$ in Iran.

In Iran, the prevalence of the major depression is higher than that what reported globally (Fallah, Farhadi, Amini, and Mohajeri, 2010). The prevalence of mild, moderate and severe depression among specific populations is $30.1 \%, 25.4 \%$, and $14.8 \%$, respectively, which is higher than global statistics (Fallah, Farhadi, Amini, and Mohajeri, 2010). So that the prevalence of mild, moderate and severe depression among specific populations estimated 1.30, 25.4\% and 14.8\%, respectively, this is higher than global statistics (Fallah, Farhadi, Amini and Mohajeri, 2010).

In 2010, the World Organization for Disease Survey has ranked the depression at the eleventh grade of disability factor, while the organization had placed it at the fifteenth rank in 1990 (Sayeq \& et al., 2012). Therefore, with the passage of time, the severity of its impact increases on society and should be paid more attention to it day by day.

Motivational and cognitive biases (orientations) are considered one of the main factors in the etiology of depression (Sadouk and Saduk, 2007).

Cognitive theories of depression emphasize that the deteriorated self-concept is the main cause of the onset and continuation of depression disorders (Beck, 1967; quoted by Moien Al-Gharbei, Nofaresti, Karamloo and Ganji, 2017); and this self-concept is a part of the schema concept, which forms the central part of Beck's cognitive theory.

According to the views and researches made by the theorist psychologists in the new branches of cognitive psychology and psychotherapy such as Yang et al., any psychological and personality problems or mental illness about how the person to know his/ her and his environment is based on the primary schemas that are formed in his mind. Primary incompatible schemas are emotional and cognitive patterns of self-harm that at the beginning of growth and development, they have been formed in the mind and repeat in the course of life and they are self 
- perpetuating; thus resist against change (Yang et al., 2007).

In examining the specific content hypothesis, Beck, Stier, and Epestin (1992) concluded that psychological states and disorders are distinguished in relation to the particular cognitive content of the individual. In this study, the noted theoretical framework is to investigate the cognitive content of Jeffrey Young's theory of primary incompatible schemas (Yang, 1994; quoted by Shahamat, 2010).

The origin of the schemas is the main emotional needs including five domains 1) a secure devotion to others, 2) autonomy, competition, and identity feeling, 3) freedom of expression of needs and emotions, 4) play and spontaneity, and 5) reasonable and self-control constraints, initial experiences of life (failing to meet initial needs, overestimate indulgence, and replicating with parents' inappropriate behavior) and emotional temperament of the child (a set of temperamental characteristics of the child that are intrinsic and distinguishing the child's primary character) (Yang, 1994; quoted by Shahamat, 2010).

Schemas arise in relation to five fundamental transformational task and they place into five groups:

First Group: Disconnection and exclusion, that its schemas are based on abandonment/instability, distrust/misconduct, emotional deprivation, defect/shame, social isolation/alienation.

Second Group: Disrupted function and autonomy (home rule), whose associated schemas are dependency/incompetence, disease vulnerability toward disease, miss self-evolution/entanglement, and failure.

Third Group: Disruptive constraints, the schemas from which originated, merit/high privilege, inadequate selfcontrol.

Forth Group: Other orientation and schemas of this domain are obedience, sacrifice, and seeking confirmation/attention desire.

Fifth Group: Excessive vigilance and inhibition, and its schemas include: negativism/pessimism, emotional inhibition, harsh / pre-critical criteria and ultimately attitude to punishment (Harris \& Katherine, 2002). Young believes that each of the symptoms of psychopathology is related to one or more primary schemas (Dilat \& et al., 200(Dilat et al., 2004). It is said in researches, primary incompatible schemas are ineffective mechanisms that directly or indirectly lead to psychological distress (Marmon \& et al., 2004; Taylor, 2005). Lamelli and Harkens (2007) achieved a meaningful relationship in a study of the symptoms of depression and anxiety and primary incompatible schemas in adolescents. In their study, they anticipated the symptoms of depression with two social isolation / alienation and sacrifice scheme. In a study to examine the relationship between parental styles perception, primary ineffective schemas and depression symptoms in young adults, they concluded that Young's schemas have a mediator or intermediary role in the relationship between perceived parental styles and depression symptoms. Among them, four most important schemes with the highest variance were: defect/shame, inadequate self-control, vulnerability, and dependency/deficiency. In a study, Marmon \& et al. (2004) found positive results about the relationship between primary incompatible schemas and feeling of reluctance.

In their studies, Kalvit \& et al. (2005) found a meaningful relationship between the symptoms of emotional disorders of depression, anxiety and anger, and primary incompatible schemas. Renick and Simons (2005) in the study about the vulnerability of adolescents to depression found that primary incompatible schemas with low social skills were important factors in increasing the vulnerability of adolescents to depression. They came to the conclusion that a number of factors associated with depression such as primary traumatic experiences, childparent interactions patterns, biological factors, and life events have been associated with failure to develop effective schemes and social skills. Halversen \& et al. (2009) studied 140 patients with clinical depression and people who were not depressed. They found a significant difference related to primary incompatible schemas. In the regression model of this study, the schemes of obedience inadequate self-control and disadvantaged constraints were important anticipator of depression symptoms. Now, considering that knowing and understanding of emotions, excitements and attitudes of oneself and others is one of the significant issues in social cognition, the identifying of how to interact of different aspects of social cognition (in this research are primary incompatible schemas) in the major depressive disorder as the most common and most costly neurotic disorder is of great importance. Therefore, according to the high cost of depression and the less costly prevention of treatment, and also considering that, despite the success of researchers and clinical experts of the cognitivebehavioral approach in the development of effective therapies for axis I disorders such as depression, many patients do not respond to this kind of treatment, and about $30 \%$ of those who feel immediately right after treatment, relapse into depression after one year; it seems necessary to take into account previous and current researches on causes and factors of depression, with a more comprehensive view of the causes and other factors involved in the emergence, stabilization and recurrence of depression as a disease that diverts a person from the field of social activities and increases desire to isolation and misunderstanding of social relationships, more researches are needed. The present study also aims to investigate the differences among the primary incompatible schemas with respect to the age range of the sisters in normal and depressed people.

\section{Method}

The present study is operational in terms of purpose and it is comparative in terms of the method. In this study 
participants included two groups;

The first group, people with the major depressive disorder who were referred to counseling and psychological services centers in Tehran for receiving psychological and psychological services.

The second group was patients' accompaniment who was similar to depressed group in terms of demographic characteristics such as gender, age, economic status and education level. Among them, two groups of 60people, depressed group 30 and common or normal group 30 people were considered. The depressed group was selected using the available method and the normal group was selected through matching with the depressed group in a classified random sampling method (in order to observe the gender ratio). For the depressed group the main conditions for entering to research were, having the major depression based on structured clinical interviews for emotional disturbances, having high school education, and fluent speaking ability. Exit criteria are also included, having concurrent psychosis disorders, personality disorders, or other disorders in form of simultaneous mental retardation (deficiency) and drug dependence. The normal group participated in a psychological interview prior to their selection, in order to confirm the absence of depression and other psychiatric disorders.

\section{Measuring Tools}

Primary incompatible schemes questionnaire-short form (YSQ-sfz)

This 75-item questionnaire was developed by Young in 1998 to evaluate 15 primary incompatible schemes. These 15 schemes are divided into five areas of cutting and exclusion, autonomy (home rule) and impaired performance, other orientation, excessive vigilance, and inhibition, and the disadvantaged constraints are in accordance with the primary developmental areas. Each question is scored on a scale of 6 degrees. If any subscale is above 25, then the schema is ineffective. Schmidt \& et al. obtained an alpha coefficient 83.0 to 96.0 for each primary incompatible schema, and the retest coefficient in the non-clinical population was between 0.5 and 0.82. Yang's study (1999) also confirmed the structure and validity of the structure of this scale. In Iran, Cronbach's Alpha has been reported 0.97 in the female population and in the male population 0.98 (Ahi, 2004). In the present study, reliability of the aforesaid questionnaire was measured with Cronbach's alpha, which was the reliability of 0.85 .

\section{Method of implementation and analysis}

After conducting clinical interviews, the primary incompatible schemas questionnaire was provided for both depressed and normal groups to answer it. Data were analyzed by multivariate analysis of variance (MANOVA) and by SPSS version 23 software.

\section{Findings}

In the table (1), the mean and standard deviation of the subscales of primary incompatible schemes are presented in the research groups.

The descriptive statistics table of the research variables in two groups with the major depressive disorder and normal people

Table 1. The mean and standard deviation of the subscales of primary incompatible schemes

\begin{tabular}{|c|c|c|c|}
\hline \multirow{2}{*}{ Variable } & \multirow{2}{*}{ Components } & Depressive Group & Normal Group \\
\hline & & mean (standard deviation) & mean (standard deviation) \\
\hline \multirow{15}{*}{ 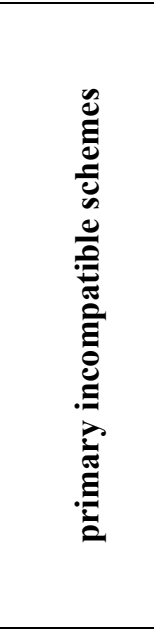 } & Emotional deprivation & $22 / 7(11 / 53)$ & $12 / 530(9 / 04)$ \\
\hline & Rejection and exclusion & $19 / 62(7 / 14)$ & $11 / 67(5 / 34)$ \\
\hline & Distrust & $19 / 02(6 / 36)$ & $9 / 44(4 / 37)$ \\
\hline & Isolation & $19 / 84(6.38)$ & $16 / 78(5 / 8)$ \\
\hline & Defect & $17 / 43(6 / 05)$ & $14 / 12(3 / 19)$ \\
\hline & Pessimistic & $19 / 16(6 / 72)$ & $10 / 08(5 / 16)$ \\
\hline & Affiliation & $7 / 87(6 / 48)$ & $9 / 44(5 / 43)$ \\
\hline & Vulnerability & $13 / 86(6 / 7)$ & $14 / 09(5 / 67)$ \\
\hline & Entangled & $14 / 15(4 / 37)$ & $13 / 89(6 / 44)$ \\
\hline & Obedience & $17 / 33(5 / 79)$ & $18 / 54(4 / 97)$ \\
\hline & Sacrifice & $11 / 09(4 / 73)$ & $11 / 99(5 / 57)$ \\
\hline & Emotional restraint & $18 / 13(7 / 53)$ & $11 / 12(4 / 89)$ \\
\hline & Implacability & $18 / 96(3 / 89)$ & $16 / 68(5 / 37)$ \\
\hline & Dignity & $14 / 22(5 / 5)$ & $15 / 92(4 / 98)$ \\
\hline & Inhibition & $15 / 98(5 / 63)$ & $17 / 73(6 / 13)$ \\
\hline
\end{tabular}

Multivariate analysis of variance (ANOVA) was used to study the difference between the primary incompatible schemes in two groups. Embac test was used to study in the covariance matrix equation assumption. 
Vol.9, No.10, 2019

The results of this test showed no significant difference in covariance matrix in any of the above variables ( $P$ $<0.005)$. The other assumption of this analysis is the homogeneity of the variance of errors, which it used the Loon test to study this assumption. The results of this test did not show any of error in the tests. Therefore, the multivariate analysis variance assumptions were used to examine the differences between the two groups in the research variables in Table (2).

Table 2. Analysis of variance to examine the differences between the two groups with the major depressive disorder and normal people

\begin{tabular}{|c|c|c|c|c|c|c|}
\hline Variables & $\begin{array}{c}\text { Sum of } \\
\text { Square Roots }\end{array}$ & $\begin{array}{c}\text { Degrees of } \\
\text { freedom }\end{array}$ & $\begin{array}{c}\text { Average } \\
\text { Squares }\end{array}$ & $\begin{array}{c}\text { F } \\
\text { Statics }\end{array}$ & $\begin{array}{c}\text { Significance } \\
\text { level }\end{array}$ & $\begin{array}{c}\text { Squat } \\
\text { Square Root }\end{array}$ \\
\hline $\begin{array}{c}\text { Emotional } \\
\text { deprivation }\end{array}$ & $593 / 33$ & 1 & $593 / 33$ & $22 / 43$ & $0 / 0005$ & $0 / 19$ \\
\hline $\begin{array}{c}\text { Rejection and } \\
\text { exclusion }\end{array}$ & $321 / 41$ & 1 & $321 / 41$ & $15 / 09$ & $0 / . .3$ & $0 / 11$ \\
\hline Distrust & $789 / 12$ & 1 & $789 / 12$ & $25 / 57$ & $0 / 0001$ & $0 / 3$ \\
\hline Isolation & $198 / 32$ & 1 & $198 / 32$ & $12 / 24$ & $0 / 007$ & $0 / 13$ \\
\hline Defect & $199 / 23$ & 1 & $199 / 23$ & $12 / 99$ & $0 / 005$ & $0 / 12$ \\
\hline Pessimistic & $551 / 29$ & 1 & $551 / 29$ & $21 / 19$ & $0 / 0001$ & $0 / 21$ \\
\hline Affiliation & $269 / 42$ & 1 & $269 / 42$ & $17 / 13$ & $0 / 0001$ & $0 / 29$ \\
\hline Vulnerability & $198 / 32$ & 1 & $198 / 32$ & $11 / 25$ & $0 / 006$ & $0 / 13$ \\
\hline Entangled & $241 / 71$ & 1 & $241 / 71$ & $14 / 95$ & $0 / 001$ & $0 / 17$ \\
\hline Obedience & $73 / 85$ & 1 & $73 / 85$ & $0 / 69$ & $0 / 44$ & - \\
\hline Sacrifice & $65 / 12$ & 1 & $65 / 12$ & $1 / 1$ & $0 / 19$ & - \\
\hline $\begin{array}{c}\text { Emotional } \\
\text { restraint }\end{array}$ & $432 / 65$ & 1 & $432 / 65$ & $14 / 33$ & $0 / 001$ & $0 / 13$ \\
\hline Implacability & $543 / 19$ & 1 & $543 / 19$ & $25 / 12$ & $0 / 0001$ & $0 / 17$ \\
\hline Dignity & $96 / 15$ & 1 & $96 / 15$ & $0 / 43$ & $0 / 23$ & - \\
\hline Inhibition & $465 / 19$ & 1 & $465 / 19$ & $18 / 86$ & $0 / 0001$ & $0 / 18$ \\
\hline Age gap & $708 / 11$ & 1 & $708 / 11$ & $23 / 78$ & $0 / 0001$ & $0 / 10$ \\
\hline As can & 1 & 1 & & \\
\hline
\end{tabular}

As can be seen in Table (2), all subscales of the primary incompatible schemes except the three subscales of sacrifice, obedience and dignity were significantly different between the two groups $(\mathrm{P}<0.05)$.

Finally, in the prediction of the major depressive disorder, a logistic regression analysis was used to assess the contribution of each of the subscales of primary incompatible schemes, the results of which are presented in Tables (3) and (4).

At first, it is showed the results of correlation matrix that among 15 sub-scales of primary incompatible schemes, emotional deprivation, rejection and exclusion, distrust, isolation, defect, pessimism, affiliation, vulnerability, entangled, emotional restraint, implacability, and inhibition are variables in which there is power predict the major depressive disorder. The coefficients of the explanation of the logistic regression model are presented in Table (3).

Table 3. The coefficients of the explanation of the logistic regression model

\begin{tabular}{|c|c|c|}
\hline Step & Coefficient of determination of Snell and Cox & The coefficient of determination of Nijil and Kirk \\
\hline 1 & $0 / 216$ & $0 / 257$ \\
\hline 2 & $0 / 246$ & 299 \\
\hline 3 & $0 / 266$ & $0 / 325$ \\
\hline 4 & $0 / 272$ & $0 / 334$ \\
\hline 5 & $0 / 277$ & $0 / 341$ \\
\hline 6 & $0 / 284$ & $0 / 350$ \\
\hline 7 & $0 / 292$ & $0 / 361$ \\
\hline 8 & $0 / 301$ & $0 / 373$ \\
\hline 9 & $0 / 313$ & $0 / 383$ \\
\hline 10 & $0 / 316$ & $0 / 388$ \\
\hline 11 & $0 / 321$ & $0 / 404$ \\
\hline 12 & $0 / 343$ & $0 / 447$ \\
\hline
\end{tabular}

As shown in Table (4), the coefficient of determination of Snell and Cox is 0.34 and the coefficient of determination of Nijal and Kirk is 0.45 . These indexes have the same interpretation as the coefficient of determination in a simple regression, that is, it represents the power to explain the dependent variable variables (people with the major depressive disorder and normal) through independent variables. In other words, the level of explanation of the dependent variable changes is represented by 12 variables, so the analysis of the data showed that 34 to $45 \%$ dependent variables are explained by these 12 variables. As shown in Table (4), this 
regression model is significantly stable for all variables $(\mathrm{P}<0.001)$.

Table 4. the results of Logistic regression analysis of variables entered in the regression model

\begin{tabular}{|l|c|c|c|c|}
\hline \multicolumn{1}{|c|}{ Variables } & Beta Coefficient & Parent Statics & Degree of Freedom & Meaningfulness \\
\hline Defect and Shame & $0 / 583$ & $29 / 92$ & 1 & $0 / 001$ \\
\hline Affiliation & $0 / 567$ & $28 / 89$ & 1 & $0 / 001$ \\
\hline Pessimistic & $0 / 553$ & $27 / 77$ & 1 & $0 / 001$ \\
\hline Emotional deprivation & $0 / 526$ & $25 / 33$ & 1 & $0 / 001$ \\
\hline Inhibition & $0 / 503$ & $22 / 14$ & 1 & $0 / 005$ \\
\hline Implacability & $0 / 485$ & $19 / 86$ & 1 & $0 / 001$ \\
\hline Entanglement & $0 / 469$ & $18 / 99$ & 1 & $0 / 002$ \\
\hline Emotional Restraint & $0 / 434$ & $18 / 37$ & 1 & $0 / 008$ \\
\hline Vulnerability & $0 / 429$ & $17 / 45$ & 1 & $0 / 0012$ \\
\hline Isolation & $0 / 425$ & $16 / 7$ & 1 & $0 / 0017$ \\
\hline Distrust & $0 / 409$ & $15 / 76$ & 1 & $0 / 005$ \\
\hline Rejection \& exclusion & $0 / 379$ & $14 / 33$ & 1 & $0 / 001$ \\
\hline Age gap & $0 / 329$ & $13 / 12$ & 1 & $0 / 00$ \\
\hline
\end{tabular}

\section{Discuss}

Concerning the difference or non-difference between the two groups in the primary schemas, the results showed that the two groups had a significant difference in all the primary incompatible schemes. The average scores of depressed people in all of the primary incompatible schemes, except for the three subscales of obedience, sacrifice, and dignity were generally was higher than those of normal people.

Therefore, the results of this study confirmed the existence of a relationship and the predictive ability of the primary incompatible schemes on the major depression. The hypothesis of this research has been meaningfully confirmed. This result is consistent with the volume of researches carried out in this regard (Harris \& Kerten (2002), Lamelli \& Harkens (2007), Kalvit \& et al. (2005), Renick Mark and Simons Ann (2005) and Halorsen \& et al. (2009)). In this among, the incompatible schema of defect / shame is a significant predictor with regard to the symptoms of the major depression. This schema is a cognitive based on the fact that the person is unwanted and bad and does not deserve to receive the love of others. This schema set in the first group that is related to disconnection and rejection. This category of schemas creates in a predictable way due to a lack of satisfaction of needs such as security and empathy (Taylor, 2005).

Depression symptoms were significantly predictable in relation to the three incompatible schemas: defect/shame, distrust/misconduct and self-incapacitation/preoccupied. On the other hand, the symptoms of depression were most closely related to the defect and shame scheme, which shows the problem in the scheme of fair treatment of oneself. The defect and shame related to the first group of schemas, namely, disconnection and rejection, which indicate that the lack of satisfaction of needs such as security and empathy is predictable in the family (Harris \& Kreten, 2002). The schema of shame indicates that one has a negative attitude toward oneself in terms of self-control and tolerance of failure, which this view is one of the important components of Beck's cognitive triangle in the etiology of depression. This result or Harris and Kreten (2002), Lamelly and Harkens (2007), Kalvit \& et al. (2005), Renick Mark and Simons Ann (2005), and Halorsen \& et al. (2009), results are consistent. Harris \& Kerten (2002) and Halorsen \& et al. (2009) in their study also identified the priority of an incompatible shame of shame/defect in predicting depression symptoms. The feeling of inadequacy and defect is a reminder of the negative view of oneself in the Beck Cognitive Triangle. Kalvit \& et al. (2005) in their study found that the role of mediators of negative automated thoughts, in relation to depression symptoms and primary incompatible schemas.

In the done logistic analysis, depression or mental health was considered as a dependent variable and variables related to primary incompatible schemas as predictive variables. The correlation between predictor variables and diagnostic function suggests that the incompatible schema of defect and shame is the predictor of depression and then the dependency, pessimism, emotional deprivation, inhibition, implacability, entanglement, emotional restraint, vulnerability and isolation, distrust, and abandonment, and exclusion were the strongest predictor of depression after the failure schema, respectively. The above variables had a positive correlation with the function. That is the greater the individual's score in each of the variables, the greater the likelihood of depression. In explaining these results, it can be pointed out that failure, defect, shame, and emotional deprivation schemas are all three components of unconditional schemas. In general, the schemas those are formed in the early stages of human evolution and often are in the center of the human cognitive structure, are unconditional beliefs about themselves and others. Schemes those shape later in the course of evolution are conditional. Unconditional schemas do not leave any loophole for person. It does not matter what the person is doing, because in any case the result is the same; an inadequate, infelicitous, dislikeable, misery person will be at 
risk and bad, and nothing can change it. Hence, those who have more abnormal unconditional schemas in them are more likely to suffer from lack of self-esteem and depression (Yang \& et al., 2007).

Also, in explaining the results of the present study, considering the age gap of individuals in the family, one can say that people who have an appropriate age gap with other people in the family have the ability to delay the satisfaction of needs and can tolerate a reasonable amount of failure. Given the fact that they have passed a healthy childhood, these people are able to postpone or revise their expectations on the basis of the economic situation but people who have less than 2 years of age gap with other children, have depression and feelings of failure due to emotional and family problems and lack of satisfaction of their psychological needs (Marmon \& et al., 2004). These people are not able to solve problems in their adolescents and they are angry with issues, and they cannot deal with problems wise because of the feeling of failure of their childhood, which it can lead to the major depression in these people (the same source).

\section{Conclusion}

The results of this study, first of all, confirm the role of the schemas in mental psychopathology. In this research, the researcher tried to study the specific cognitive content associated with each of the above disorders, which cognitive content was examined in the framework of primary incompatible schemas. The results obtained in this study are an admission for in-depth study of the specific cognitive content of each disorder. This will be an important step in explaining and clarifying the disorders, and on the other hand it will have important allusions in the field of treatment. In this regard, it seems that this domain requires an accurate and separate examination of each disorder in order to explain them and to map out the primary incompatible schemas involved in the continuation of symptoms.

Consequently, any scientific work that deals with the recognition of human beings and human behaviors has certain limitations, since the tool for collecting information is man-made so it cannot be codified by a complex rule. Psychological researches and therefore the present research is not an exception to this rule. Among the methodological limitations of this research, self-report tools can be mentioned.

However, in this research, a structured clinical interview was also used, but because ultimately the final measurement tool was the self-report scale, the caution is essential in extending the results of the study. Investigating other effective factors in etiology of this disorder, such as attachment style and mood, may also lead to more precise follow-up studies, especially for patients who leave the treatment halfway.

\section{Sources}

Ahi, Qasem; Mohammadifar, Mohammad Ali; Besharat, Mohammad Ali (2007), Reliability and validity of the short form of Young's Schema Questionnaire, Volume 37, No. 3, pp. 5-20

Shahamat, Fatemeh (2010), Prediction of general health symptoms (physicalization, anxiety and depression) based on primary incompatible schemas. Modern Psychological Researches (Psychology of Tabriz University), Vol. 5, No. 20, pp. 103-124.

Bakhshipour Abbas, Vojoudi Babak, Mahmoud Aliloo, Majid Abdi, Reza. (2016). Effectiveness of Integrated Fractal Therapy in Reducing Symptons of The major Depressive Disorders, The Journal of Thoughts and Behavior, Volume 11, No. 41, pp. 67-87.

Rezaei Mehdi, Ghadampour Ezatollah, Rezaei Mazaher, Kazemi Reza. (2015), the Effectiveness of Emotional Schema Therapy (EST) on the Disclosure and Severity of Depression in Patients with The major Depression, Journal of Clinical Psychology, 2011 (Issue 4), pp. 45-59. 